\title{
An Optimal Algorithm for Midcourse Guidance Law Under Wind Disturbance
}

\author{
Sy-Hieu Nguyen ${ }^{1}$, Dinh-Dung Nguyen ${ }^{2,3,}$, \\ ${ }^{1}$ Department of Missile, Faculty of Control Engineering, Le Quy Don Technical University, Hanoi, Vietnam \\ ${ }^{2}$ Department of Aircraft System Design, Faculty of Aerospace Engineering, Le Quy Don Technical University, Hanoi, Vietnam \\ ${ }^{3}$ Department Aeronautics, Naval Architecture and Railway Vehicles, Faculty of Transportation Engineering and Vehicle Engineering, \\ Budapest University of Technology and Economics, Budapest, Hungary
}

\section{Email address:}

Nguyensyhieu30@gmail.com (Sy-Hieu N.), ddnguyen@vrht.bme.hu (Dinh-Dung N.)

${ }^{*}$ Corresponding author

\section{To cite this article:}

Sy-Hieu Nguyen, Dinh-Dung Nguyen. An Optimal Algorithm for Midcourse Guidance Law Under Wind Disturbance. American Journal of Aerospace Engineering. Vol. 7, No. 2, 2020, pp. 6-14. doi: 10.11648/j.ajae.20200702.11

Received: March 22, 2020; Accepted: April 8, 2020; Published: December 31, 2020

\begin{abstract}
The guidance laws are commonly designed to yield as small a miss distance as possible, harmonious with the missile's acceleration capability. In recent decades, the concept of optimized guidance law is well understood in applications where information concerning the target range and line-of-sight angle is available. Researchers' efforts have been continually made to apply modern control theory to conventional and adaptive autopilot designs, even though the classical theory is still applicable to autopilots. It can be noted that it is desirable to perform a detailed computer-aided feasibility study within the context of a realistic missile-target engagement model. Development and evaluation of guidance and control laws for simplified missile-target engagement scenarios are extended and adapted to the air-to-air missile situation and implemented in a complete three-dimensional engagement model. Thus, this study proposed a computational method for constructing an optimal midcourse guidance law, which is based on the optimal control theory and initial boundary conditions. This proposed guidance law is derived from an optimal control theory with the boundary conditions such as allowed relative distance between missile and target at the final time, low line-of-sight rate. A numerical simulation verifies the performance of this guidance law with the impact of harmonic wind. The simulation results demonstrate that the quality of effectiveness as well as the applicability of this proposed algorithm.
\end{abstract}

Keywords: Optimal Control, Optimal Guidance Law, Midcourse Phase, Wind Disturbance

\section{Introduction}

The guided missiles consist of aerodynamic guided missiles, which use an aerodynamic lift to control its direction of flight. An aerodynamic guided missile can be defined as an aerospace vehicle, with varying guidance capabilities, that is self-propelled through the atmosphere to inflict damage on a designated target. Two common types of guided missiles that create a threat to aircraft are the air-to-air (AA), or air-intercept missiles (AIM), and the surface-to-air (SAM). The AA and SAM missiles are launched from interceptor fighter aircraft and employing various guidance techniques. Surface-to-air missiles can be launched from land or sea-based platforms.
Conventional proportional navigation systems have been improved with time-variable filtering, and the design process has been enhanced with automatic computer approaches. Several studied have been taken in the past to investigate, evaluate, and improve the proportional navigation problem [1, 8-12]. With online Kalman estimation for filtering noisy radar data and optimal control gains, the guidance systems have been developed in performances. These approaches are commonly designed to yield as small a miss distance as possible, consistent with the missile's acceleration capability.

In recent decades, the concept of optimized guidance law is well understood in applications where information concerning the target range and line-of-sight angle is available. Researchers' efforts have been continually made to apply 
modern control theory to conventional and adaptive autopilot designs, even though the classical theory is still applicable to autopilots.

Optimal control and estimation theory have been commonly used in the design of advanced guidance systems from the late 1960s [2-7, 13-16]. Such approaches have been used to develop tracking algorithms that extract the maximum amount of information about a target trajectory. This information used to optimize the directed missile toward the selected destination. Compared to general guidance and control techniques, the advantages of the optimized system are most significant against maneuverable airborne targets, where target acceleration information and rapid guidance system response time are required to achieve acceptable accuracy, in minimum time. Besides, to solve highly non-linear flight control problems, neural network algorithms, and fuzzy logic theory have been developed, which is motivated by the demand to deal with non-linear flight control and performance robustness problems.

It can be noted that it is desirable to perform a detailed computer-aided feasibility study within the context of a realistic missile-target engagement model. The guidance and control laws have been developed and evaluated for simplified missile-target engagement scenarios, which must be extended and adapted to the air-to-air missile situation and then implemented in a complete three-dimensional engagement model.

In reference [1], by considering the desired impact angle without violating the field-of-view limit, the authors studied a two-stage pure proportional navigation guidance law. Majumder et al. investigated a near-optimal solution in real-time for air-to-air engagement [2]. This solution is used to solve the midcourse guidance problem in real-time for air-to-air engagement. The authors also presented recent developments in this field.

In reference [3], the authors proposed an optimal midcourse guidance law with flight path angle and lead angle constraints to reach a circular target area. This guidance law was derived by applied an optimal control theory, which minimizes control energy weighted by a power of a range-to-go. However, in this research, the target was considered as a stationary target because it moves so slowly. For that target, Zhang et al. proposed a novel closed-form guidance law with impact time and impact angle constraints [4]. This guidance law takes the missile's normal acceleration as the control command directly. By simplifying missile dynamics under small heading error approximation, Chen and Wang derived an optimal guidance law with impact angle constraint against a stationary target [5]. An impact time requirement is achieved by adding a feedback controller to the obtained optimal guidance law.

By solving an optimal control problem minimizing the energy cost function weighted by a power of range-to-go, Park proposed an optimal guidance law with terminal angle constraint at the end of the midcourse phase [6]. In reference [7], considering the final velocity vector constraint, an optimal terminal guidance law was developed for exoatmospheric interception using the optimal control theory. By taking the gravity difference model in this approach, the proposed guidance law requires much less fuel than the traditional ones in the exoatmospheric interception. Various missile guidance laws-based optimal theory has been developed, and their performances have been verified in several aspects, including accuracy, robustness, and efficiency [17-22].

In recent years, the combined guidance laws have been applied widely for air-to-air missiles. These guidance laws have several advantages, such as the significant distance of attack, high accuracy, sizeable initial look-angle.

In the literature review, however, the targets move so slowly or stationary. Therefore, the parameters of the target cannot be introduced in the geometric dynamic equations that represent the relationship between the missile and target. Then, by applying the optimal control theory, the analytics guidance law can be natural to synthesize. For maneuver targets, the guidance law is based on the proportional navigation guidance law with impact angle and impact time [5, 6, 19-21]. This guidance law, however, is not compared to the optimal one. So, in this study, an optimal guidance law is proposed for a missile to attack a maneuvering target. This guidance law is derived from the optimal control theory with the boundary conditions such as allowed relative distance between missile and destination at the final time, low line-of-sight rate.

The rest of this paper is as follows. In section 2, based on the dynamic missile model, a state equation system is deduced. An optimal midcourse guidance law is also presented in this section, which is based on the boundary conditions at the final time and optimal control theory that minimizes a range of weighted control energy. The simulation results and investigation of the proposed guidance law with the influence of wind are given in section 3, concluding the paper will be presented in section 4 .

\section{Computation of an Optimal Midcourse Guidance Law}

\subsection{Equation of Motion}

The desired trajectory of a missile is shown in Figure 1.

Where, $V_{m}$ and $\psi_{m}$ are velocity and flight path angle of the missile, respectively; $(\mathrm{x}, \mathrm{z})$ is the position of the missile; $\left(x_{m}^{*}, z_{m}^{*}\right)$ is the desired position of the missile; $\psi_{m}^{*}$ is the desired flight path angle of the missile; $h$ is the instant deviation that is defined as a relative distance from the target, which is perpendicular to the velocity vector.

The equations of motion in the $x_{0} x_{1} z_{l}$ coordinate as follows:

$$
\left\{\begin{array}{l}
\Delta \dot{\psi}=\frac{g}{V_{m}} n_{z} \\
\dot{x}_{1}=V_{m} \cos \Delta \psi \\
\dot{z}_{1}=V_{m} \sin \Delta \psi
\end{array}\right.
$$


We assume that $x_{m}$ is a variable, then, $z_{m}=f\left(x_{I}\right)$ is the desired trajectory in the coordinate's $x_{0} x_{1} z_{l}$. The function $f\left(x_{1}\right)$ must ensure rectilinear asymptote with the straight line $x_{0} x_{1}$.

The function $f\left(x_{1}\right)$ is described in detail as follows:

A function which represents the transition from $O x z$ coordinate to $x_{0} x_{1} z_{l}$ coordinate is given in (2).

$$
z=K \frac{x-x_{0}}{\sigma^{2}} e^{-\frac{\left(x-x_{0}\right)^{2}}{2 \sigma^{2}}}
$$

where, $O x$ is the horizontal asymptote of the function $z$;

$$
z=0 \text { when } x-x_{0}=0 \text {; and } \mathrm{z}=z_{\max }=\frac{K}{\sigma} e^{-\frac{1}{2}} \text { when } x-x_{0}=\sigma \text {. }
$$

We aim to find the values $K$ and $\sigma$ so that the graph of the function $z$ passes through the point $\left(x_{1}, a\right)$ and reaches the maximum value at $\left(x_{2}, b\right)$. Where, $x_{1}, x_{2}, a$, and $b$ are known values. Using the division method, we get a solution $x^{*}$ from which we find $K$ and $\sigma$ satisfy the requirement. The first and second derivatives of (2) are given as (3) and (4):

$$
\begin{gathered}
\sigma=\frac{x_{2}-x_{1}}{1-x^{*}} ; K=b e^{\frac{1}{2}} \sigma \\
f_{x}=\frac{\partial z}{\partial x}=\frac{K}{\sigma^{2}} e^{-\frac{\left(x-x_{0}\right)^{2}}{2 \sigma^{2}}}\left(1-\left(\frac{x-x_{0}}{\sigma}\right)^{2}\right) \\
f_{x x}=-\frac{K}{\sigma^{2}} \frac{x-x_{0}}{\sigma^{2}}\left(3-\frac{\left(x-x_{0}\right)^{2}}{\sigma^{2}}\right) e^{-\frac{\left(x-x_{0}\right)^{2}}{2 \sigma^{2}}}
\end{gathered}
$$

It can be seen from the Figure 1 that the deviation between a missile trajectory and the desired one is determined as follows: $\Delta h=h-z_{m}=z_{1}-z_{m}$ two-sides derivation and combination with (1) we have:

$$
\Delta \dot{h}=V_{m} \sqrt{1+f_{x}^{2}} \sin \left(\Delta \psi-\psi_{m}\right)
$$

$\psi_{m}$ is the desired flight path angle at the time t.

Let $\Delta \bar{\psi}=\Delta \psi-\psi_{m}$ is the bias between the flight path angle and the desired one. Two-sides derivative is:

$$
\Delta \dot{\bar{\psi}}=\Delta \dot{\psi}-\dot{\psi}_{m}
$$

Combining with the first equation of the (1), we have:

$$
\left\{\begin{array}{l}
\Delta \dot{\bar{\psi}}=\frac{g}{V_{m}} n_{z}-\dot{\psi}_{m} \\
\Delta \dot{h}=V_{m} \sqrt{1+f_{x}^{2}} \sin (\Delta \bar{\psi})
\end{array}\right.
$$

where, $n_{z}$ is the normal overload factor.

$$
\text { Let } n_{z m}=\sqrt{1+f_{x}^{2}}\left(n_{z}-\frac{V_{m}}{g} \dot{\psi}_{m}\right) ; \bar{V}_{m}=V_{m} \sqrt{1+f_{x}^{2}}
$$

We get:

$$
\left\{\begin{array}{l}
\Delta \dot{\bar{\psi}}=\frac{g}{\bar{V}_{m}} n_{z m} \\
\Delta \dot{h}=\bar{V}_{m} \sin (\Delta \bar{\psi})
\end{array}\right.
$$

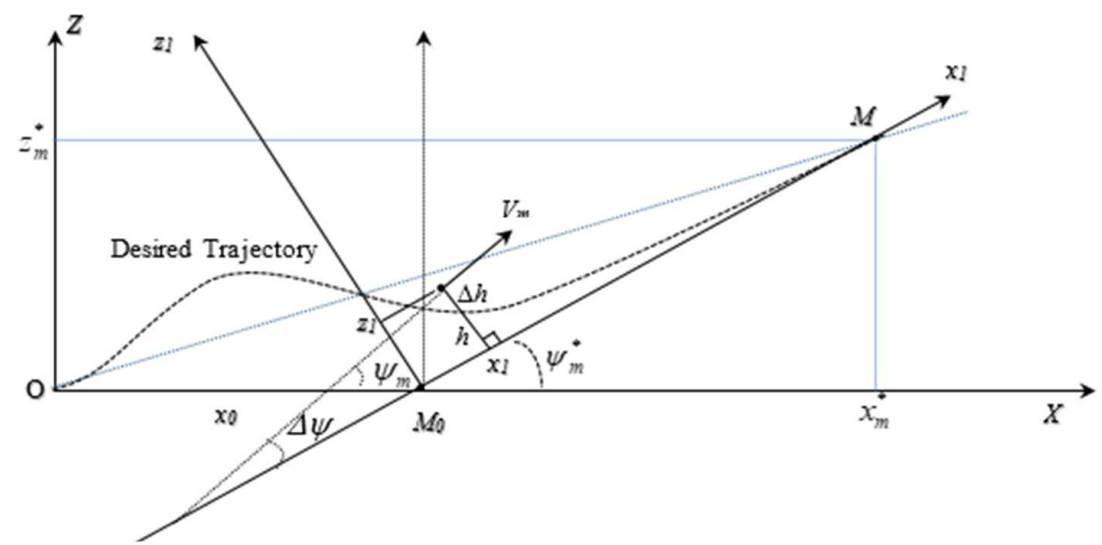

Figure 1. The desired trajectory of a missile.

\subsection{Computation of an Optimal Midcourse Guidance Law}

Let us consider the cost function is as follows:

$$
\begin{gathered}
J=\frac{1}{2} \rho_{1} \Delta \bar{\psi}_{t_{f}}^{2}+\frac{1}{2} \rho_{2} \Delta h_{t_{f}}^{2}+\frac{1}{2} \int_{t_{o}}^{t_{f}} k n_{z 0}^{2} d t \rightarrow \min \\
G=\frac{1}{2}\left[\begin{array}{c}
\Delta \bar{\psi} \\
\Delta h
\end{array}\right]_{t_{f}}^{T}\left[P^{f}\right]_{2 \times 2}\left[\begin{array}{c}
\Delta \bar{\psi} \\
\Delta h
\end{array}\right]_{t_{f}} ; P^{f}=\left[\begin{array}{cc}
\rho_{1} & 0 \\
0 & \rho_{2}
\end{array}\right]
\end{gathered}
$$

$P^{f}$ is the symmetric matrix.

From (7) and (8) the Hamiltonian function is as follows:

$$
H=\lambda_{\Delta \bar{\psi}} \frac{g}{\bar{V}_{m}} n_{z m}+\lambda_{\Delta h} \bar{V}_{m} \sin \Delta \bar{\psi}+\frac{1}{2} k n_{z m}^{2}
$$

Co-state variables are defined as the following: 


$$
\left\{\begin{array}{l}
\frac{d \lambda_{\Delta \bar{\psi}}}{d t}=-\frac{\partial H}{\partial \Delta \bar{\psi}}=-\lambda_{\Delta h} \bar{V}_{m} \cos \Delta \psi \\
\frac{d \lambda_{\Delta h}}{d t}=-\frac{\partial H}{\partial h}=0
\end{array}\right.
$$

The expression determines the optimal solution $\partial H / \partial n_{z_{m}}=0$, we obtain:

$$
n_{z m}=-\frac{g}{k} \frac{1}{\bar{V}_{m}} \lambda_{\Delta \bar{\psi}}
$$

From (9), the Teminant function is: $G=\frac{1}{2} \rho_{1} \Delta \bar{\psi}_{t_{f}}^{2}+\frac{1}{2} \rho_{2} \Delta h_{t_{f}}^{2}$, we have converted boundary conditions:

$$
\left\{\begin{array}{l}
\left.\lambda \overline{\Delta \bar{\psi}}\right|_{t_{f}}=\left.\frac{\partial G}{\partial \Delta \bar{\psi}}\right|_{t_{f}}=\left.\rho_{1} \Delta \bar{\psi}\right|_{t_{f}} \\
\left.\lambda_{\Delta h}\right|_{t_{f}}=\left.\frac{\partial G}{\partial \Delta h}\right|_{t_{f}}=\left.\rho_{2} \Delta h\right|_{t_{f}}
\end{array}\right.
$$

into a matrix form:

$$
\left[\begin{array}{l}
\lambda_{\Delta \bar{\psi}} \\
\lambda_{\Delta h}
\end{array}\right]_{t_{f}}=\left[P^{f}\right]\left[\begin{array}{l}
\Delta \bar{\psi} \\
\Delta h
\end{array}\right]_{t_{f}} ; P^{f}=P^{f}=\left[\begin{array}{cc}
\rho_{1} & 0 \\
0 & \rho_{2}
\end{array}\right]
$$

The Equation (8) is rearranged in a matrix form as the following:

$$
\left[\begin{array}{c}
\Delta \dot{\bar{\psi}} \\
\Delta \dot{h}
\end{array}\right]=\left[\begin{array}{cc}
0 & 0 \\
\bar{V}_{m} & 0
\end{array}\right]\left[\begin{array}{l}
\Delta \bar{\psi} \\
\Delta h
\end{array}\right]+\left[\begin{array}{c}
\frac{g}{\bar{V}_{m}} \\
0
\end{array}\right] n_{z m}
$$

where, $\sin \Delta \bar{\psi} \approx \Delta \bar{\psi}$

The dynamics of missile is given:

$$
\dot{x}=A^{(11)} x+B u
$$

where,

$$
A^{(11)}=\left[\begin{array}{cc}
0 & 0 \\
\bar{V}_{m} & 0
\end{array}\right], B=\left[\begin{array}{c}
\frac{g}{V_{m}} \\
0
\end{array}\right], x=\left[\begin{array}{c}
\Delta \bar{\psi} \\
\Delta h
\end{array}\right], u=n_{z m}
$$

From (11), we can rewrite in the following form:

$$
\left[\begin{array}{l}
\dot{\lambda}_{\Delta \bar{\psi}} \\
\dot{\lambda}_{\Delta h}
\end{array}\right]=\left[\begin{array}{cc}
0 & \bar{V}_{m} \cos \Delta \bar{\psi} \\
0 & 0
\end{array}\right]\left[\begin{array}{l}
\lambda_{\Delta \bar{\psi}} \\
\lambda_{\Delta h}
\end{array}\right]
$$

where, $\dot{\lambda}=A^{(22)} \lambda$

$$
A^{(22)}=\left[\begin{array}{cc}
0 & \bar{V}_{m} \cos \Delta \bar{\psi} \\
0 & 0
\end{array}\right] ; \lambda=\left[\begin{array}{l}
\lambda_{\Delta \bar{\psi}} \\
\lambda_{\Delta h}
\end{array}\right]
$$

From (12) and (14) we have:

$$
\dot{x}=A^{(11)} x-B \frac{g}{k} \frac{1}{\bar{V}_{m}}\left[\begin{array}{ll}
1 & 0
\end{array}\right] \lambda
$$

We can rewrite in the following form:

$$
\dot{x}=A^{(11)} x+A^{(12)} \lambda
$$

where, $A^{(12)}=-\frac{1}{k}\left(\frac{g}{\bar{V}_{m}}\right)^{2}\left[\begin{array}{ll}1 & 0 \\ 0 & 0\end{array}\right]$

And from (15) and (16) we obtain:

$$
\left[\begin{array}{c}
\dot{x} \\
\dot{\lambda}
\end{array}\right]=\left[\begin{array}{ll}
A^{(11)} & A^{(12)} \\
A^{(21)} & A^{(22)}
\end{array}\right]\left[\begin{array}{l}
x \\
\lambda
\end{array}\right]
$$

where, $A^{(21)}=[0]_{2 \times 2}$

From (13), the states at the final time are determined as follows:

$$
\left[\begin{array}{l}
x \\
\lambda
\end{array}\right]_{t_{f}}=\left[\begin{array}{c}
I_{2 \times 2} \\
P^{f}
\end{array}\right] x_{t_{f}}
$$

The solution of (17) is $\left[\begin{array}{l}x \\ \lambda\end{array}\right]=\Phi\left(t_{f}-t\right)\left[\begin{array}{l}x \\ \lambda\end{array}\right]_{t_{f}}$

Substituting into (18), we have:

$$
\left[\begin{array}{l}
x \\
\lambda
\end{array}\right]=\left[\begin{array}{ll}
\Phi_{11}\left(t_{f}-t\right) & \Phi_{12}\left(t_{f}-t\right) \\
\Phi_{21}\left(t_{f}-t\right) & \Phi_{22}\left(t_{f}-t\right)
\end{array}\right]\left[\begin{array}{c}
I_{5 \times 5} \\
P^{f}
\end{array}\right] x_{t_{f}}
$$

From (19), we get:

$$
\begin{gathered}
x=\left(\Phi_{11}+\Phi_{12} P^{f}\right) x_{t f} \Rightarrow x_{t_{f}}=\left(\Phi_{11}+\Phi_{12} P^{f}\right)^{-1} x \\
\lambda=\left(\Phi_{21}+\Phi_{22} P^{f}\right) x_{t_{f}}=P^{T}(t) x
\end{gathered}
$$

where, $P^{T}(t)=\left(\Phi_{21}+\Phi_{22} P^{f}\right)\left(\Phi_{11}+\Phi_{12} P^{f}\right)^{-1}$

From (13) and (20), we obtain boundary conditions:

$$
P^{T}\left(t_{f}\right)=P^{f}
$$

Two-sides derivative of (20) we get:

$$
\dot{\lambda}=P^{T}(t) \dot{x}+\dot{P}^{T}(t) x
$$

From (15), (16), (20), and (22) we get:

$$
\begin{aligned}
& A^{(22)} P^{T}(t) x=P^{T}(t)\left(A^{(11)} x+A^{(12)} P^{T}(t) x\right)+\dot{P}^{T}(t) x \\
& \dot{P}(t)=P(t)\left(A^{(22)}\right)^{T}-\left(A^{(11)}\right)^{T} P(t)-P(t) A^{(12)} P(t)
\end{aligned}
$$




$$
\dot{P}(t)=\left[\begin{array}{cc}
\bar{V}_{m}\left(\cos \Delta \bar{\psi} P_{12}-P_{21}\right) \frac{1}{k}\left(\frac{g}{\bar{V}_{m}}\right)^{2} P_{11}^{2} & -\bar{V}_{m} P_{22}+\frac{1}{k}\left(\frac{g}{\bar{V}_{m}}\right)^{2} P_{11} P_{12} \\
\bar{V}_{m} \cos \Delta \bar{\psi} P_{22}+\frac{1}{k}\left(\frac{g}{\bar{V}_{m}}\right)^{2} P_{21} P_{11} & \frac{1}{k}\left(\frac{g}{\bar{V}_{m}}\right)^{2} P_{21} P_{12}
\end{array}\right]
$$

From (12) and (20) an optimal midcourse guidance law is determined as follows:

$$
n_{z m}=-\frac{g}{k} \frac{1}{\bar{V}_{m}}\left[\begin{array}{ll}
1 & 0
\end{array}\right] P^{T}(t) x
$$

To find $\mathrm{P}(\mathrm{t})$, it is necessary to solve the Riccati equation (24) with boundary conditions (21). However, this is very difficult because the (24) has no analytic solution. Therefore, here we find the approximate solution by adding constraints on the quality criteria of the system.

From (25), we have:

$$
n_{z m}=-\frac{g}{k} \frac{1}{\bar{V}_{m}}\left(P_{11} \Delta \bar{\psi}+P_{21} \Delta h\right)
$$

Let $k_{1}=\frac{g}{k} \frac{1}{\bar{V}_{m}} P_{11} ; k_{2}=\frac{g}{k} \frac{1}{\bar{V}_{m}} P_{21}$, we get:

$$
n_{z m}=-k_{1} \Delta \bar{\psi}-k_{2} \Delta h
$$

So, instead of finding $P_{11}, P_{2 l}$, we find $k_{1}$ và $k_{2}$; Substituting (26) into (7), we have:

$$
\Delta \dot{\bar{\psi}}=-\frac{g}{V_{m}}\left(k_{1} \Delta \bar{\psi}+k_{2} \Delta h\right)
$$

A two-sided derivation of (27) is given:

$$
\Delta \ddot{\bar{\psi}}=-\frac{g}{\bar{V}_{m}}\left(k_{1} \Delta \dot{\bar{\psi}}+k_{2} \Delta \dot{h}\right)
$$

Let $y_{1}=\Delta \bar{\psi} ; y_{2}=\Delta \dot{\bar{\psi}}$, the (28) is rearranged as the following:

$$
\left\{\begin{array}{l}
\dot{y}_{1}=y_{2} \\
\dot{y}_{2}=-\frac{g}{\bar{V}_{m}} k_{1} y_{2}-g k_{2} y_{1}
\end{array} \text { Or }\left[\begin{array}{l}
\dot{y}_{1} \\
\dot{y}_{2}
\end{array}\right]=\left[\begin{array}{cc}
0 & 1 \\
-g k_{2} & -\frac{g}{\bar{V}_{m}} k_{1}
\end{array}\right]\left[\begin{array}{l}
y_{1} \\
y_{2}
\end{array}\right]\right.
$$

The characteristic equation of the equation system (29) is followed:

$$
\begin{gathered}
\alpha^{2}+\frac{g}{\bar{V}_{m}} k_{1} \alpha+g k_{2}=0 \\
\alpha_{1,2}=\frac{-\frac{g}{\bar{V}_{m}} k_{1} \pm \sqrt{\left(\frac{g}{\bar{V}_{m}} k_{1}\right)^{2}-4 g k_{2}}}{2}
\end{gathered}
$$

The general solution of (28) is given:

$$
y_{1}=\Delta \bar{\psi}=c_{1} e^{\alpha_{1} t}+c_{2} e^{\alpha_{2} t}
$$

Equation (31) is the general solution form of the homogeneous (28). In order to errors in the stable mode become zero, just satisfy conditions $\alpha_{1}<0$ and $\alpha_{2}<0$. To avoid over-tuning, $\alpha_{1}$ and $\alpha_{2}$ are real numbers, which means that:

$$
\left(\frac{g}{\bar{V}_{m}} k_{1}\right)^{2}>4 g k_{2} \Rightarrow k_{1}>2 \bar{V}_{m} \sqrt{\frac{k_{2}}{g}} ; k_{2}>0
$$

Because $k_{1}>0$ and $k_{2}>0$, then $\alpha_{1}>\alpha_{2}$. Therefore, if $\alpha_{1}<0$, it will be sure that $\alpha_{2}<0$. Besides, we have: $-1 / \alpha_{1}=\tau$, where $\tau$ is a time constant.

We add two constraints to the system. The first constraint is that the control law must ensure that the time constant is not higher than the allowed time constant.

Then, we obtain:

$$
-\frac{-\frac{g}{\bar{V}_{m}} k_{1}+\sqrt{\left(\frac{g}{\bar{V}_{m}} k_{1}\right)^{2}-4 g k_{2}}}{2}=\frac{1}{T_{C P}}
$$

where, $\mathrm{T}_{\mathrm{CP}}$ is the allowed time constant.

Therefore, we get:

$$
k_{2}=\frac{1}{\bar{V}_{m} T_{C P}}\left(k_{1}-\frac{\bar{V}_{m}}{g T_{C P}}\right)
$$

The second constraint is that a normal overload (the value of the control signal) is not higher than the allowed overload $\left(\mathrm{n}_{\mathrm{zmCP}}\right)$, which means that $\left|\mathrm{n}_{\mathrm{zm}}\right| \leq \mathrm{n}_{\mathrm{zmCP}}$.

Integrating with (26), we get:

$$
n_{z m C P}=k_{1} \Delta \bar{\psi}_{\max }+k_{2} \Delta h_{\max }
$$

where, $\Delta \bar{\psi}_{\max }, \Delta h_{\max }$ are the maximum value of bias of flight path angle and instant deviation, respectively. Then,

$$
k_{1}=\frac{n_{z m C P}+\frac{1}{g T_{C P}^{2}} \Delta h_{\max }}{\frac{1}{\bar{V}_{m} T_{C P}} \Delta h_{\max }+\Delta \bar{\psi}_{\max }}
$$

Substituting $k_{l}$ into (33), we get:

$$
k_{2}=\frac{n_{z m C P}+\frac{\bar{V}_{m}}{g T_{C P}} \Delta \bar{\psi}_{\max }}{\Delta h_{\max }+\bar{V}_{m} T_{C P} \Delta \bar{\psi}_{\max }}
$$


From (7) and (26), we obtain:

$$
n_{z}=-\frac{k_{1} \Delta \bar{\psi}+k_{2} \Delta h}{\sqrt{1+f_{x}^{2}}}+\frac{\bar{V}_{m}}{g} \dot{\psi}_{m}
$$

So, the equation (36) is the optimal midcourse guidance law with the coefficients are determined as (34) and (35); and $f_{x}, \dot{\psi}_{m}$ are calculated as (3) and (5).

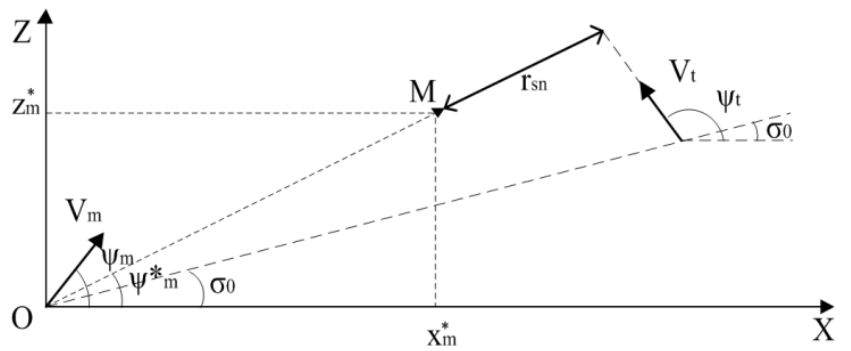

Figure 2. Determining a desired line-of-sight angle.

\subsection{Determining the Desired Line-of-Sight Angle at the Final Time}

Based on the Figure 2, we obtain:

$$
\left\{\begin{array}{l}
\left(t_{s n} V_{m}+r_{s n}\right) \sin \Delta \psi=t_{s n} V_{t} \sin \Delta \psi_{t} \\
\left(t_{s n} V_{m}+r_{s n}\right) \cos \Delta \psi=r_{0}+t_{s n} V_{t} \cos \Delta \psi_{t}
\end{array}\right.
$$

Where, $r$ is a relative distance between a missile and a target, then $r\left(t_{f}\right)=r_{s n}$, and $r\left(t_{0}\right)=r_{0} ; t_{f}$ is a final time; $\mathrm{t}_{\mathrm{sn}}$ is a self-navigation time after finishing the midcourse phase.

Solving this equations system, we get:

$$
\begin{gathered}
t_{s n}=\frac{r_{0}}{V_{m}} \frac{-\left(k_{r}-k_{V} \cos \Delta \psi_{t}\right)+\sqrt{\left(k_{r}-k_{V} \cos \Delta \psi_{t}\right)^{2}+\left(1-k_{V}^{2}\right)\left(1-k_{r}^{2}\right)}}{\left(1-k_{V}^{2}\right)} \\
\psi_{m}^{*}=\arcsin \left(\frac{t_{s n} V_{t}}{t_{s n} V_{m}+r_{t d}} \sin \Delta \psi_{t}\right)+\sigma_{0} \\
z_{m}^{*}=t_{s n} V_{m}\left(\frac{t_{s n} V_{t}}{t_{s n} V_{m}+r_{s n}} \sin \Delta \psi_{t}\right) \\
x_{m}^{*}=t_{s n} V_{m} \frac{r_{0}+t_{s n} V_{t}}{r_{s n}+t_{s n} V_{m}} \cos \Delta \psi_{t}
\end{gathered}
$$

However, since the missile trajectory is a high curve, we have to calculate the amount of compensation. Therefore, the desired line-of-sight (LOS) angle compensated by the missile's curved trajectory is given as follows:

$$
\psi_{m}^{*}=\arcsin \left(\frac{t_{s n} V_{t}}{t_{s n} V_{m}+r_{s n}} \sin \Delta \psi_{t}\right)+\sigma_{0}+\psi_{c}
$$

where, $\Psi_{c}$ is a compensation of the flight path angle.

The compensation of the flight path angle is calculated as follows:

$$
\sin \psi_{c} \approx V_{t} t_{e} / r_{s n}
$$

where, $t_{e}$ is the time error when the missile reaches the engagement point; $r_{s n}$ is a relative distance between the missile and target at the final time.

To eliminate slippage, this compensation is given:

$$
\sin \psi_{c}=-\frac{V_{t}}{V_{m}} \sin \left(\Delta \psi-\Delta \psi_{t}\right)
$$

\subsection{A wind Turbulence Model}

It can be noted that wind turbulence can be modeled in several forms such as disturbance models according to the horizontal wind field and harmonized wind field. In this paper, we investigate the influence of the harmonized wind field in the horizontal plane to the quality of the proposed guidance law.

The harmonized wind model is given as follows:

$$
\left\{\begin{array}{l}
V_{\mathrm{W} x_{0}}=10 \sin \left(2 \pi t / T_{W}\right) \\
V_{\mathrm{Wz}_{0}}=10 \sin \left(2 \pi t / T_{W}+\varphi\right)
\end{array}\right.
$$

\section{Simulation Results and Discussion}

In this work, numerical simulations were designed for evaluating the quality of effectiveness as well as the applicability of this proposed guidance law.

In the simulation experiments, the parameters of a missile and target are given in Table 1.

Table 1. The parameters of a missile and target.

\begin{tabular}{lll}
\hline Parameter & & Value \\
\hline Velocity of missile & $V_{m}$ & $900 \mathrm{~m} / \mathrm{s}$ \\
Overload factor & $\left|n_{m}\right|$ & $\leq 30$ \\
Line-of-sight angle & $\sigma_{0}$ & atan $(30 / 31)$ \\
Initial missile position & $\left(x_{m 0}, z_{m 0}\right)$ & $(0,0) \mathrm{m}$ \\
Target position & $\left(x_{t}, z_{t}\right)$ & $(31.000,31.0000) \mathrm{m}$ \\
Relative distance between the missile and target at the final time & $r_{s n}$ & $10.000 \mathrm{~m}$ \\
Velocity of target & $V_{t}$ & $300 \mathrm{~m} / \mathrm{s}$ \\
Path angle of target & $\Psi_{t}$ & $135^{\circ}$ \\
Velocity of wind & $W_{0}$ & $10 \mathrm{~m} / \mathrm{s}$ \\
\hline
\end{tabular}


In order to investigate the impact of wind on the proposed guidance law, we design four cases to do numerical simulation. In this study, we assume that the wind is harmonic wind with amplitude $\mathrm{W}_{0}=10 \mathrm{~m} / \mathrm{s}$, period $\mathrm{T}_{\mathrm{w}}=10 \mathrm{~s}$, and the time of occurrence $T \in[5 ; 15] \mathrm{s}$. The direction angle of wind compared to $\mathrm{Oz}$ axis are $\pi / 6, \pi / 3, \pi / 2$ corresponding to case 2 , 3 , and 4 . The mathematical model of wind in four cases is given in the table 2 .

Table 2. The mathematical model of wind.

\begin{tabular}{llll}
\hline Case 1 & Case 2 & Case 3 & Case 4 \\
\hline No wind & $\left\{\begin{array}{l}V_{\mathrm{W} x_{0}}=10 \sin \left(2 \pi t / T_{\mathrm{W}}\right) \\
V_{\mathrm{W} z_{0}}=10 \sin \left(\frac{2 \pi t}{T_{W}}+\frac{\pi}{6}\right)\end{array}\right.$ & $\left\{\begin{array}{l}V_{\mathrm{W} x_{0}}=10 \sin \left(2 \pi t / T_{\mathrm{W}}\right) \\
V_{\mathrm{W} z_{0}}=10 \sin \left(\frac{2 \pi t}{T_{W}}+\frac{\pi}{3}\right)\end{array}\right.$ & $\left\{\begin{array}{l}V_{\mathrm{W} x_{0}}=10 \sin \left(2 \pi t / T_{\mathrm{W}}\right) \\
V_{\mathrm{W} z_{0}}=10 \sin \left(\frac{2 \pi t}{T_{W}}+\frac{\pi}{2}\right)\end{array}\right.$ \\
\hline
\end{tabular}

The Figure 3 shows the missile trajectory and target trajectory in four cases. It can be seen that the missile trajectories in four cases are the same. However, when the harmonic wind is introduced, there is a bias compared with the case that does not consider the wind (see Figure 4).

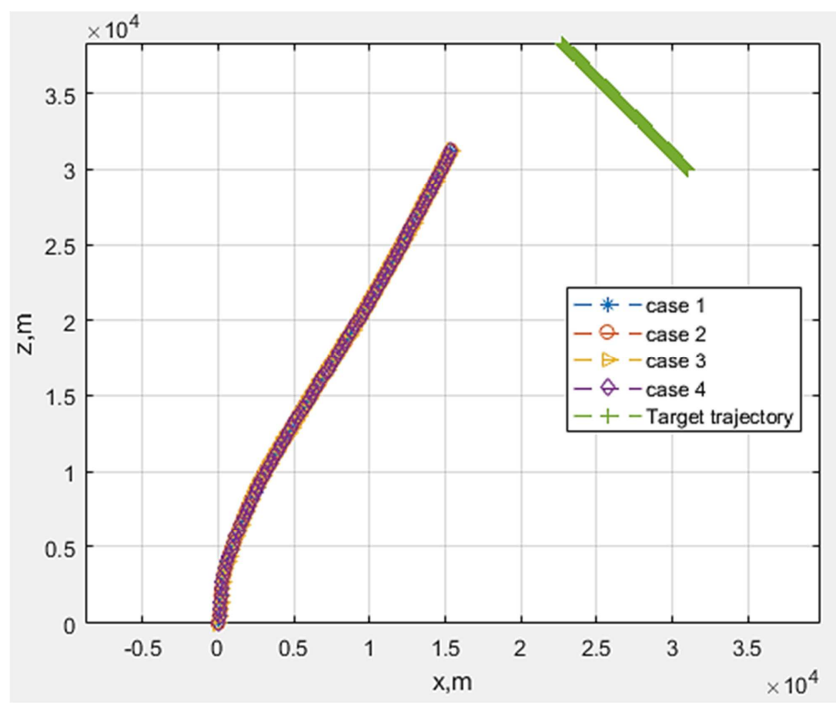

Figure 3. The missile trajectory and target trajectory.

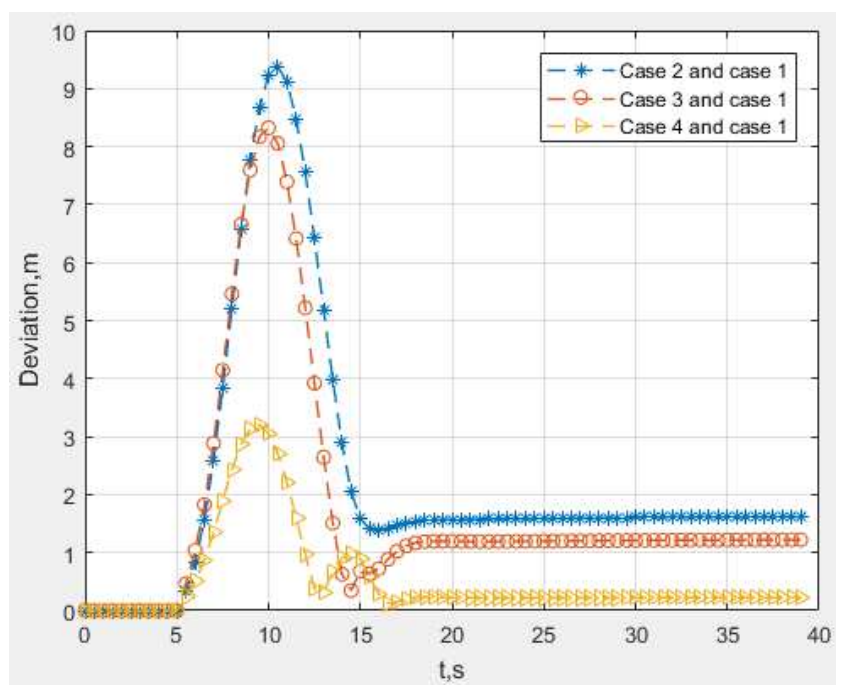

Figure 4. The difference of missile trajectories between four cases.

The overload factor and its difference in the four cases are shown in Figure 5 and Figure 6. When the wind occurs, there was a bias of overload factor $\left(\Delta \mathrm{n}_{\mathrm{m}}=1.3\right)$. However, this bias will be disappeared when the wind is finished.

Figure 7 and Figure 8 represent the instant deviation and its bias. In Figure 7, as expected, the instant deviation goes to zero. Also, it becomes significant in the period that wind is introduced, and quickly reduces to instantaneous value over time as wind is disappeared.

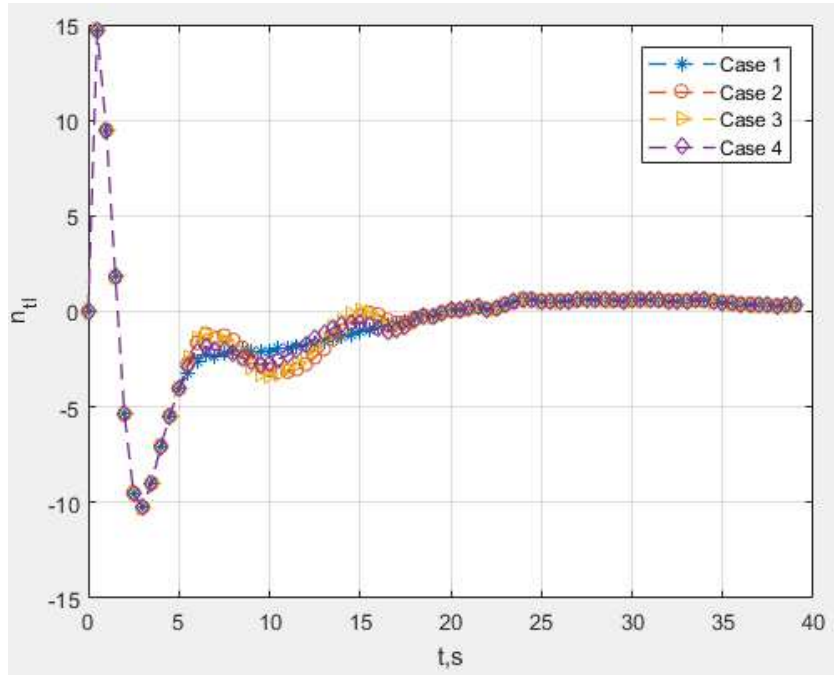

Figure 5. The overload factor.

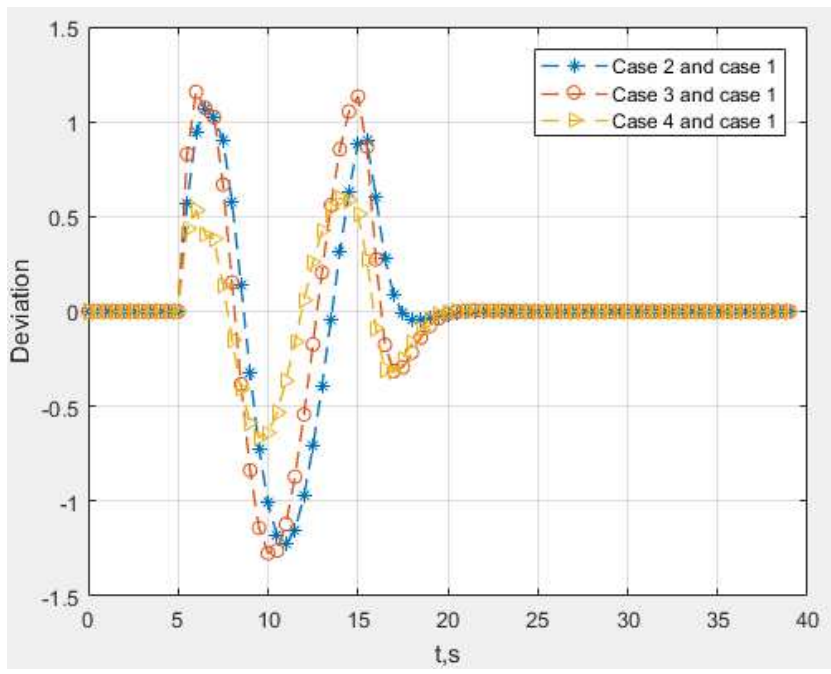

Figure 6. The difference of overload factor between four case. 


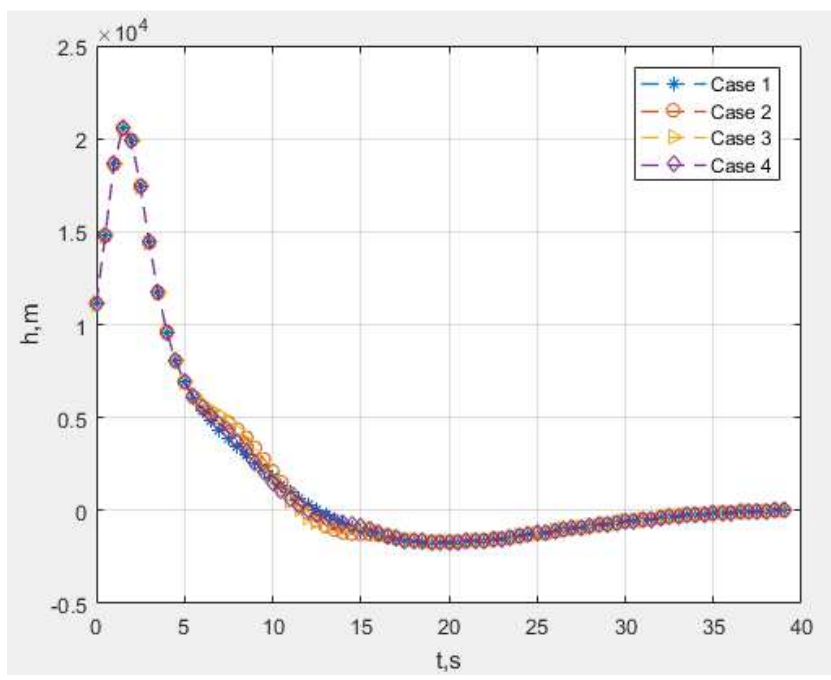

Figure 7. The instant deviation.

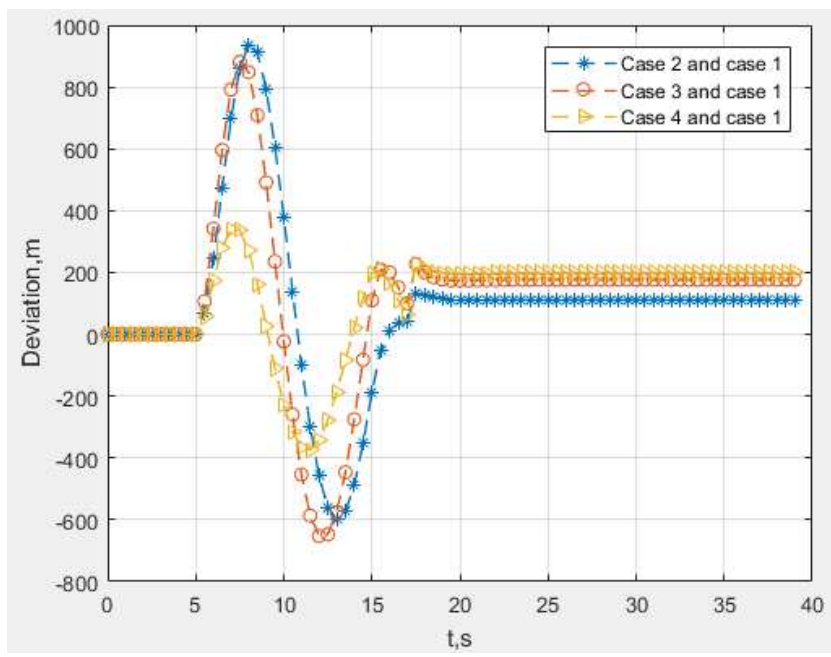

Figure 8. The difference of instant deviation.

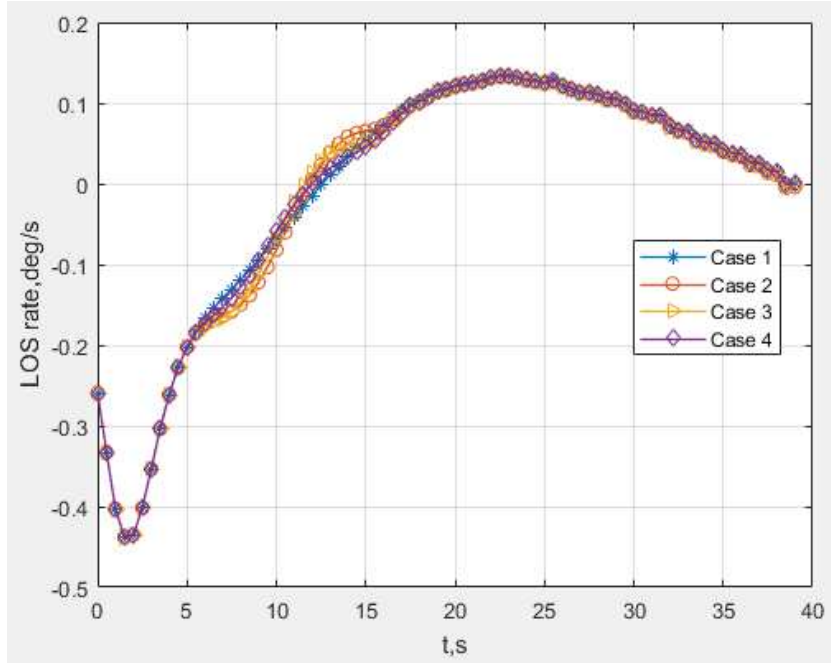

Figure 9. The line-of-sight rate.

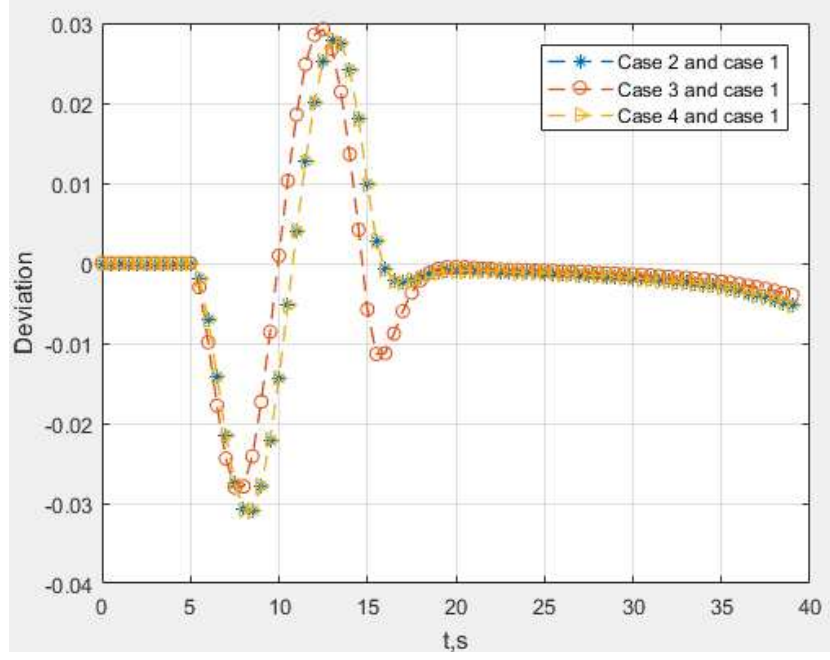

Figure 10. The difference of the line-of-sight rate.

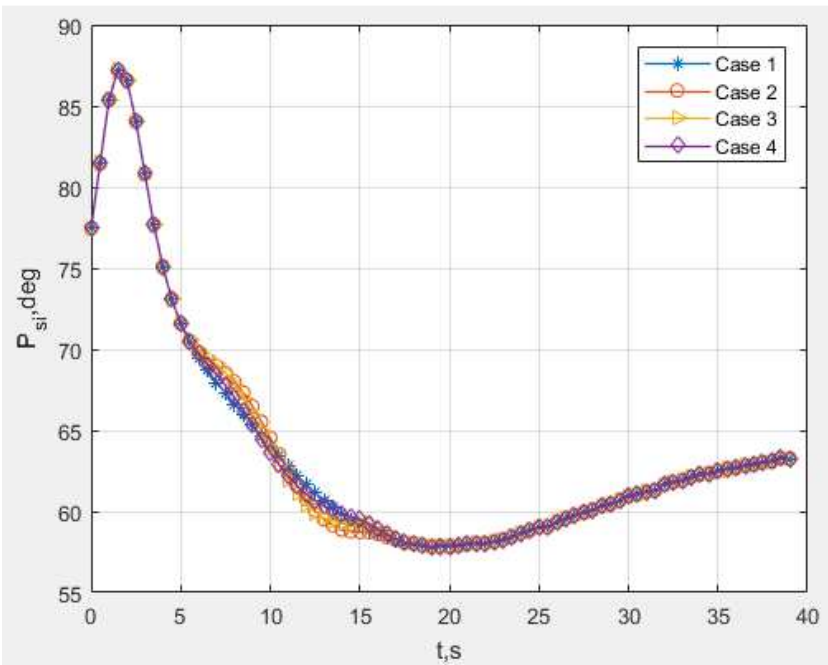

Figure 11. The heading angle of the missile.

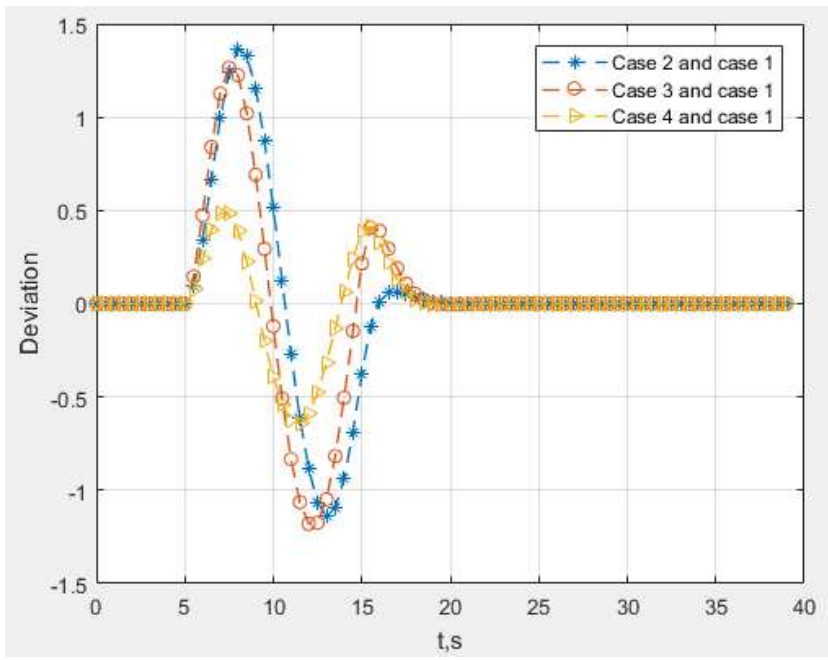

Figure 12. The difference of the heading angle.

The LOS rate and its difference between four cases are shown in Figure 9 and Figure 10. There is a deviation of the LOS rate at the final time between two cases: one considers a harmonic wind, whereas the other does not consider it. 
However, it should be noted that this deviation is so small, $\Delta \dot{\sigma} \approx-0,005 \mathrm{deg} / \mathrm{s}$.

The heading angle and its difference between four cases are shown in Figure 11 and Figure 12. It can be seen that the heading angle reaches the desired one even there is a disturbance wind.

\section{Conclusion}

The parameters of slowly or stationary targets cannot be introduced in the geometric dynamic equations that represent the relationship between the missile and target. Then, the analytics guidance law can be synthesized by using the optimal control theory. However, for maneuver targets, the guidance law based on the proportional navigation is not compared to the optimal one.

So, an optimal midcourse guidance law is presented in this paper, which is based on the optimal control theory that minimizes a range of weighted control energy with initial boundary conditions. The simulation results show that the constraint requirements are satisfied at the final time, such as low LOS rate, overload factor, instant deviation. By introducing the harmonic wind, the simulation results provide the evaluations of the quality of effectiveness as well as the applicability of this proposed guidance law.

\section{References}

[1] Ratnoo, Ashwini. "Analysis of two-stage proportional navigation with heading constraints." Journal of Guidance, Control, and Dynamics 39.1 (2015): 156-164. doi: 10.2514/1G001262.

[2] Majumder, S. B., et al. "Practical aspects of optimal midcourse guidance for air-to-air engagement." IFAC-PapersOnLine 49.1 (2016): 591-596. Doi: 10.1016/j.ifacol.2016.03.119.

[3] Jeon, Byoung-Ju, Bong-Gyun Park, and Min-Jea Tahk. "Optimal midcourse guidance law with flight path angle and lead angle constraints to reach circular target area." IFAC

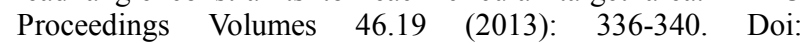
10.3182/20130902-5-DE-2040.00090.

[4] Zhang, Youan, Guoxin Ma, and Aili Liu. "Guidance law with impact time and impact angle constraints." Chinese Journal of

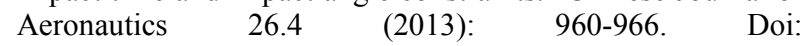
10.1016/j.cja.2013.04.037.

[5] Chen, Xiaotian, and Jinzhi Wang. "Optimal control based guidance law to control both impact time and impact angle." Aerospace Science and Technology 84 (2019): 454-463. Doi: 10.1016/j.ast.2018.10.036.

[6] Park, bong-gyun. "Optimal impact angle constrained guidance with the seekers lock-on condition." Journal of the Korean Society for Industrial and Applied Mathematics 19.3 (2015): 289-303. Doi: 10.12941/jksiam.2015.19.289.

[7] Yu, Wenbin, et al. "Optimal terminal guidance for exoatmospheric interception." Chinese Journal of Aeronautics 29.4 (2016): 1052-1064. Doi: 10.1016/j.cja.2016.04.019.

[8] M. Guelman. A qualitative study of proportional navigation. IEEE Transactions on Aerospace and Electronic Systems, no. 4, pp. $637-643,1971$.

[9] S. Ghosh, D. Ghose, and S. Raha. Capturability analysis of a 3-D retro-PN guidance law for higher speed nonmaneuvering targets. IEEE Transactions on Control Systems Technology, vol. 22, no. 5, pp. 1864-1874, 2013.

[10] N. Dhananjay, D. Ghose, and M. S. Bhat. Capturability of a geometric guidance law in relative velocity space. IEEE Transactions on Control Systems Technology, vol. 17, no. 1, pp. 111-122, 2008.

[11] S. Lee, S. Ann, N. Cho, and Y. Kim. Capturability of Guidance Laws for Interception of Nonmaneuvering Target with Field-of-View Limit. Journal of Guidance, Control, and Dynamics, vol. 42, no. 4, pp. 869-884, 2019.

[12] D.-M. Ma, J.-K. Shiau, and I. Ho. Missile guidance using dual-mode seeker. Journal of Emerging Trends in Engineering and Applied Sciences, vol. 2, no. 4, pp. 636-642, 2011.

[13] M. Balesdent, N. Bérend, P. Dépincé, and A. Chriette. Multidisciplinary design optimization of multi-stage launch vehicle using flight phases decomposition. International Journal for Simulation and Multidisciplinary Design Optimization, vol. 4, no. 3-4, pp. 117-125, 2010.

[14] E. Civek and M. K. Özgören, "Space Launch Vehicle Design with Simultaneous Optimization of Thrust Profile and Trajectory," in AIAA SPACE and Astronautics Forum and Exposition, p. 5333.

[15] S.-M. Hong, M.-G. Seo, S.-W. Shim, M.-J. Tahk, and C.-H. Lee. Sensitivity analysis on weight and trajectory optimization results for multistage guided missile. IFAC-PapersOnLine, vol. 49, no. 17, pp. 23-27, 2016.

[16] S.-M. Hong and M.-J. Tahk, "Stage optimization of multi-stage anti-air missile using co-evolutionary augmented Lagrangian method," in 2017 25th Mediterranean Conference on Control and Automation (MED), pp. 1257-1262: IEEE.

[17] H. Cho, C.-K. Ryoo, A. Tsourdos, and B. White. Optimal impact angle control guidance law based on linearization about collision triangle. Journal of Guidance, Control, and Dynamics, vol. 37, no. 3, pp. 958-964, 2014.

[18] S.-M. Hong, D.-Y. Lee, and M.-J. Tahk. Stage Optimization of Anti-Air Missiles Considering Guidance Laws. IFAC-PapersOnLine, vol. 52, no. 12, pp. 227-231, 2019/01/01/ 2019.

[19] K. S. Erer and R. Tekin. Impact time and angle control based on constrained optimal solutions. Journal of Guidance, Control, and Dynamics, pp. 2448-2454, 2016.

[20] S. R. Kumar and D. Ghose. Impact time guidance for large heading errors using sliding mode control. IEEE Transactions on Aerospace and Electronic Systems, vol. 51, no. 4, pp. 3123-3138, 2015.

[21] R. Tekin, K. S. Erer, and F. Holzapfel. Control of impact time with increased robustness via feedback linearization. Journal of Guidance, Control, and Dynamics, vol. 39, no. 7, pp. 1682-1689, 2016.

[22] S. He, W. Wang, D. Lin, and H. Lei. Consensus-based two-stage salvo attack guidance. IEEE Transactions on Aerospace and Electronic Systems, vol. 54, no. 3, pp. 1555-1566, 2017. 